\title{
Dengue virus pathogenesis in mouse central nervous system: studies on host response to dengue virus infection Juliano Bordignon ${ }^{1}$, Christian Probst ${ }^{1}$, Ana Luiza P Mosimann ${ }^{1}$, Vanessa Stella1 ${ }^{1}$ Daniela Pavoni1 ${ }^{1}$, Greg Buck², Paul Fawcett ${ }^{2}$, Silvio M Zanata ${ }^{3}$, Lucia Noronha ${ }^{4}$, Marco Krieger ${ }^{1}$ and Claudia Nunes Duarte dos Santos*1
}

\author{
Address: ${ }^{1}$ Instituto Carlos Chagas/Fiocruz, Curitiba, Paraná, Brazil, ${ }^{2}$ Virginia Commonwealth University, Richmond, VA, USA, ${ }^{3}$ Universidade \\ Federal do Paraná/UFPR, Curitiba, Paraná, Brazil and ${ }^{4}$ Pontifícia Universidade Católica do Paraná/PUCPR, Curitiba, Paraná, Brazil \\ Email: Claudia Nunes Duarte dos Santos* - clsantos@fiocruz.br \\ * Corresponding author
}

from Infectious diseases of the nervous system: pathogenesis and worldwide impact

Paris, France. 10-13 September 2008

Published: 23 September 2008

BMC Proceedings 2008, 2(Suppl I):P8

This abstract is available from: http://www.biomedcentral.com/I753-656I/2/SI/P8

(c) 2008 Bordignon et al; licensee BioMed Central Ltd.

Dengue virus (DENV) causes a self-limiting fever (DF) or severe hemorrhagic fever/shock-syndrome (DHF/DSS). Recently, clinical profile of DENV infection is changing, and neurological manifestations are becoming frequent. We previously demonstrated that mutations on E and NS3 proteins may account for DENV neurovirulence for mice. To validate the involvement of the observed mutations in the appearance of neurovirulent viral phenotypes, we constructed cDNA infectious clones encompassing the observed mutations. Aiming to determine host response to infection with neurovirulent and parental strains of DENV-1, we used a Mus musculus biochip (Virginia Commonwealth University) encompassing whole mouse genome. Newborn Swiss mice were infected intracerebraly with $8.000 \mathrm{ffu}_{\mathrm{C} 636}$ of FGA/89 (parental strain), FGA/NA a5c (neurovirulent strain) and mock. Animals infected with FGA/NA a5c show clinical signs of encephalitis around 9 days post-infection (dpi) and succumb to death (13.1 dpi \pm 2.2$)$, in contrast with animals infected with FGA/89 and mock. Kinetics of infection showed higher levels of viral RNA and progeny in FGA/NA a5c infected animals at $9 \mathrm{dpi}$. Microarray analyzes were carried out at $5,6,7$ and 8 dpi in central nervous system (CNS) of mock, FGA/89 and FGA/NA a5c infected animals. Bioinformatic analysis revealed 149 genes up-regulated in CNS infection by DENV-1, at higher levels in animals infected with FGA/ $\mathrm{NA}$ a5c. Analysis with the software Ingenuity Pathways Systems (IPA) showed that the main pathways modulated by DENV infection in the mouse CNS are IFN signaling, antigen presentation, complement cascades and protein ubiquitination. Additionally, 15 genes were selected by in silico analyses; of special interest are four genes encoding chemokines with chemoattractant activity, that are up-regulated following infection with the neurovirulent virus compared to parental virus. Nowadays we are performing the biological characterization of the selected genes and pathways.

\section{Financial support}

Fundação Araucária, Fiocruz and CNPq. 